\title{
Can vinegar supplementation acutely influence appetite and glycaemic response?
}

\author{
J. Darzi, G. S. Frost, C. Cooke, L. Johnson, V. Martin, J. Pickard and M. D. Robertson \\ Faculty of Health \& Medical Sciences, University of Surrey, Guildford GU2 7XH, UK
}

Previous intervention studies have reported the oral provision of the SCFA propionate and acetate induce satiety ${ }^{(1-2)}$. However, effects on actual intake were not measured, visual analogue scales (VAS) accepted as the best method to subjectively assess appetite ${ }^{(3)}$ were not used, the taste effects of the SCFA (a potential confounder in appetite research) were not accounted for and the study duration was short (120 min postprandially). The present study therefore aimed to address these methodological weaknesses.

Sixteen unrestrained eaters (three male, thirteen female) aged 22.2 (SD 3.0) years were recruited to this randomised single-blind twoway cross-over study. Participants consumed a standard breakfast alongside a drink containing $25 \mathrm{~g}$ white wine vinegar or a drink without added vinegar (PL). Effects on appetite were assessed subjectively using VAS and assessed quantitatively by providing a preweighed ad libitum pasta meal $3 \mathrm{~h}$ postprandially. Capillary blood samples were regularly collected by fingerprick to monitor the postprandial glycaemic response.

Vinegar treatment significantly reduced subjective appetite ratings for the desire to eat $(P=0.043)$ and hunger $(P=0.063)$ and increased fullness $(P=0.002)$ and also nausea $(P=0.002)$ when compared with PL $180 \mathrm{~min}$ area under the curve. In addition, quantitative measures of appetite were significantly influenced, with a mean intake of the pasta meal following PL and vinegar of 3401 (SD 1004 ) kJ and 2774 (sD 883$) \mathrm{kJ}$ respectively $(P=0.048, n 15)$. Vinegar treatment was also found to significantly lower the glycaemic response for the first $120 \min (P=0.022)$.
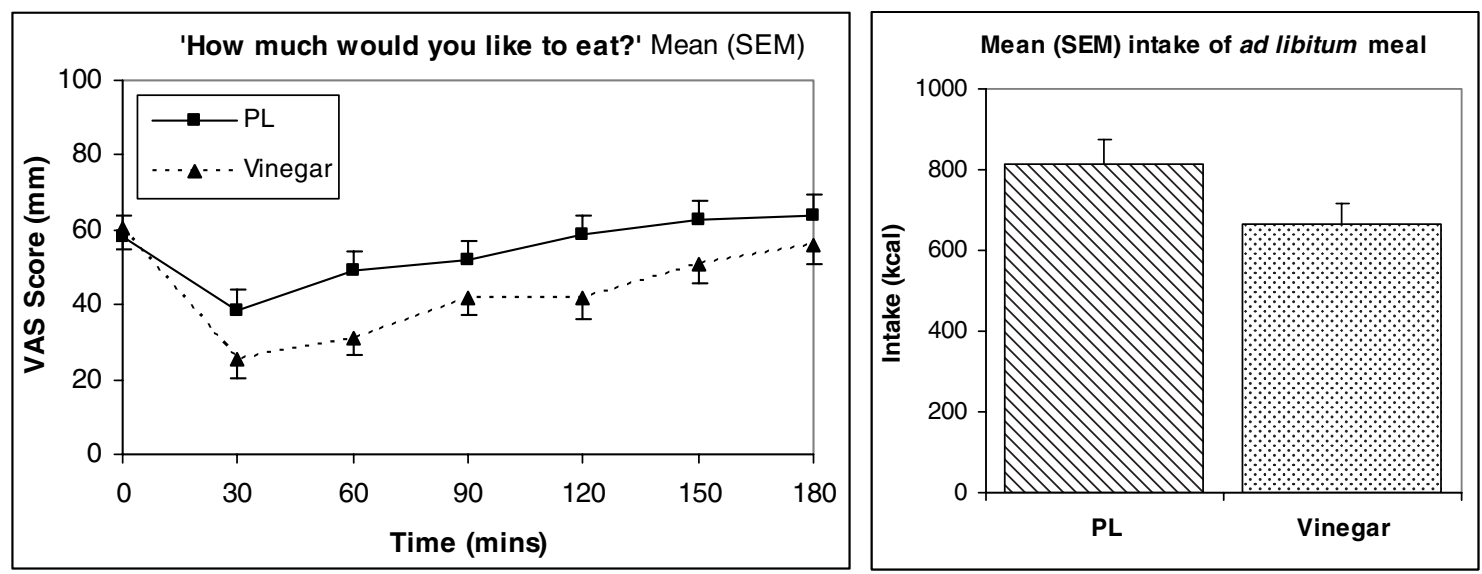

In conclusion, the findings from the present study suggest that vinegar influences appetite and blunts the glycaemic response, possibly as a result of increased nausea and the reduced palatability of the vinegar-containing product. These findings support a role for oral provision of SCFA to reduce appetite, in agreement with previous studies ${ }^{(1-2)}$.

A follow-up study is underway to investigate the orosensory effects of vinegar on appetite and metabolic response using the modified sham-feeding technique.

1. Ostman E, Granfeldt Y, Persson L et al. (2005) Eur J Clin Nutr 59, 983-988.

2. Liljeberg HG, Lonner CH \& Bjorck IM (1995) J Nut 125, 1503-1511.

3. Flint A, Raben A, Blundell JE et al. (2002) Int J Obes Relat Metab Disord 24, 38-48. 